\title{
La formación doctoral y su impacto académico-social en el desarrollo local
}

\author{
Matos Columbié Zulema de la Caridad ${ }^{1}$, Pastrana Corral Susana Angélica ${ }^{2}$, \\ Matos Columbié Ceila ${ }^{3}$ \\ ${ }^{1}$ Profesor Titular - Investigador Titular. Doctor en Ciencias Pedagógicas. Jefe de Dpto. Formación de Grados Científicos. Universidad de \\ Guantánamo, Cuba. \\ ${ }^{2}$ Profesor Titular - Investigador. Doctor en Administración Pública. Dpto. Ciencias Sociales. Campus Caborca.Universidad de Sonora, \\ México. \\ Investigador Agregado. Profesor Titular. Doctor en Ciencias Pedagógicas. Instituto Central de Ciencias Pedagógicas de Cuba. Profesor \\ Invitado de la Universidad de Guantánamo.
}

\section{Resumen}

La presente investigación pretende valorar el impacto académico-social para el desarrollo local que tiene en la actualidad el proceso de formación doctoral de los profesionales de diferentes ramas del saber, tanto en la provincia de Guantánamo, Cuba como en el Estado de Sonora, México. Se diseñó primeramente un estudio empírico en el que se aplicaron diferentes métodos y técnicas de investigación educativa en diferentes unidades muestrales (32 doctorantes de Ciencias Pedagógicas, profesores, tutores, oponentes, directivos y otros) y pudieron determinarse las regularidades que caracterizaron este proceso. Se ofrece un modelo pedagógico de formación doctoral que integra sistémicamente la formación teórico-metodológica y la investigativa a partir de desarrollar un proceso investigativo para la tesis que responda desde el aporte teórico-práctico de la ciencia, a los acuciantes problemas que se diagnostican en la práctica a la solución de los acuciantes problemas que se diagnostican en la práctica para contribuir al desarrollo local.

Palabras clave: formación doctoral, formación teórico-metodológica, formación investigativa.

\section{Doctoral training and its academic-social impact on local development}

\begin{abstract}
The present investigation dims to analize the social academic impacted for the local development that has actually in the process of de doctoral formation of the profession of the different branches of knowledge as in Guantánamo, Cuba province and in the state of Sonora, México.

It was designes as an empiric study in the wich applies different methods and investigation techniques of the education in the different units shown, doctoral students, teacher, tutors, opponents, managers and others and they coold determine the regulations the characterize this process.

A pedagogical model of doctorate formation that includes that sistematically the methodological and theoretical formation and the investigative starting with the development of the investigative process for the thesis, that responds since the practical theoretical contribution of the science, of the pressing problems that are didanose in the practice.
\end{abstract}

Keywords: doctoral training, theoretical-methodological training, research training.

*Autor para envío de correspondencia: Calle Calixto García Num 763. Entre Prado y Jesús del Sol. Guantánamo. CP. 95100. Cuba. E-mail: zulema@cug.co.cu.; zulematosco@gmail.com. E-mail: mcoronado@uabc.mx

(c) 2019 Editorial UNISON — URN. Derechos reservados. 


\section{INTRODUCCIÓN}

\author{
"En la ciencia no hay calzadas reales y \\ quien aspire a remontar sus luminosas cumbres \\ tiene que estar dispuesto a escalar la montaña por senderos \\ escabrosos" \\ Karl Marx.
}

Actualmente la formación doctoral se considera una de áreas de resultados clave de mayor significación y relevancia en las universidades, está considerada una de las figuras esenciales de la Educación de Postgrado, que tiene la misión de formar un doctor en una determinada 'parea del conocimiento competente y capaz propiciar la aplicación consciente de los adelantos científico-tecnológicos alcanzados, en función del desarrollo local de los territorios, toda vez que se proponen respuestas contextualizadas a los problemas científicos detectados.

En muchos países la Educación de Posgrado está considerada el más alto nivel de la educación Superior, por eso, la formación doctoral se constituye en una de las estructuras de la formación académica donde se preparan integralmente los cuadros científicos de las universidades y de sus respectivos países, de ahí que los egresados de un proceso de formación doctoral, a partir del desarrollo científico-tecnológico alcanzado, deben desarrollar y poseer una preparación teórico, metodológica e investigativa que le posibilite la transformación se su realidad en consonancia con las exigencias sociales.

Es por eso que urge buscar vías y las mejores alternativas para formar un doctor en ciencias competente y comprometido con la realidad científica y social, que enarbole desde la ciencia modos de actuación profesional e investigativa que ponderen su carrera, que trasmitan soluciones bien pensadas y organizadas para en el menor tiempo posible lograr las transformaciones de un estado inicial a un estado deseado en su área de conocimiento

En relación con lo anteriormente planteado y a partir de un estudio exploratorio, complementado con la descripción se concluye que la formación doctoral en las Universidades de Guantánamo y Sonora discurre desde un modelo de formación que estipula la solución de problemas a partir del banco de problemas existente, que se relacionen directamente con las principales exigencias de desarrollo económico-social, es un proceso que emerge desde los propios departamentos docentes con sus respectivas sesiones científicas, y que tiene como centro el proceso investigativo que desarrollan los doctorantes, guiados por el tutor y donde el proyecto de investigación es una entidad necesaria para el cumplimiento de las tareas investigativas.
En el contexto Latinoamericano y caribeño, la sociedad del conocimiento necesita con urgencia respuestas concretas a sus múltiples problemas y necesidades, por eso la investigación científica debe proporcionar respuestas a dichos problemas, "(...) siendo la Universidad el espacio ideal para el desarrollo de una investigación de calidad y para la formación de investigadores, que pueden y deben jugar un papel fundamental en el proceso de cambio de modelo productivo y en la generación y transferencia de conocimiento", de acuerdo con R. Duran Rill; et al (2018)

Es una realidad que los doctores deben participar activamente en el proceso de transformación del conocimiento que se genera en bienestar para la sociedad y de su área de conocimiento, lo que justifica que el modelo de formación doctoral que se preconiza en la universidad debe estar sustentado en los siguientes principios:

La orientación del doctorado en el que se articula desde la superación investigación el pregrado y postgrado, la superación profesional, con las unidades docentes, laboratorios sociales y entidades laborales (empresas, organismos, instituciones, instituciones) y de la producción y los servicios.

La formación doctoral toral centrado en la sociedad en colaboración con organizaciones sociales implicadas y conectada a proyectos de investigación más desarrollo más innovación $(\mathrm{i}+\mathrm{D}+\mathrm{I})$ con salida profesional en la producción y los servicios en función del desarrollo local.

El logro de mayor reconocimiento social a la formación doctoral como expresión de la excelencia académica y profesional para la solución de problemas que afectan a la sociedad.

Concretar desde la investigación los ejes de desarrollo estratégico de la sociedad hasta el 2030

\section{MATERIALES Y MÉTODOS}

Para la recopilación de la información necesaria en esta investigación, se decidió realizar un estudio de tipo descriptivo (R. Hernández Sampieri; et al, 2010), ya que se buscó identificar y describir las características de un proceso que aglutina un grupo de profesionales que se encuentran en la formación doctoral. A su vez, la investigación califica de tipo exploratorio (Narváez, 2009), ya que la literatura científica consultada y seleccionada para la fundamentación científica del tema reveló que existen muchos modelos de formación doctoral, sin embargo, se precisa de un referente teórico para la investigación. 
Sobre este particular, se corresponde que la valoración sobre la formación doctoral y su impacto académico-social desde la vinculación superación - investigación es un fenómeno conocido que precisa de contextualización y desarrollarse atendiendo a las características físico-geográficas, socioeconómicas y culturales del ecosistema de donde proceden los doctorantes y hacia dónde van los principales aportes (C. Matos Columbié, y Z. Matos Columbié, 2018); Z. Matos Columbié; et al, 2019)

En la presente investigación, se optó por realizar un estudio documental para analizar los principales documentos que ofrecen las bases legales y científicas de la formación doctoral (CNGC, 2017; R. Duran Rill ; et al, 2017; F. E. Robas Díaz, 2018; C. Matos Columbié, Z. Matos Columbié, 2019), posteriormente se elaboró una guía de observación a actividades formativas en la Escuela de Formación Doctoral para valorar el desempeño de profesores, tutores, doctorantes y resumir las principales regularidades que tipifican el proceso de formación doctoral en de la Universidad de Guantánamo, tal y como se ilustra en la tabla 1.

Tabla 1. Guía de observación a actividades formativas en la Escuela de Formación Doctoral para valorar el desempeño de profesores, tutores y doctorantes

\begin{tabular}{|lcc|}
\hline No & Actividades realizadas & Cantidad \\
\hline 1 & Cuestionario a doctorantes cubanos & 32 \\
\hline 2 & Conferencias & 16 \\
\hline 3 & Consultorias & 45 \\
\hline 4 & Asesorías & 32 \\
\hline 5 & Entrevista a especialistas de la Escuela de & 32 \\
\hline & Formación Doctoral & \\
\hline 6 & $\begin{array}{c}\text { Entrevista a especialistas del campus } \\
\text { Caborca de la UNISON con doctorantes } \\
\text { en la Escuela de Formación Doctoral }\end{array}$ & 2 \\
\hline
\end{tabular}

Fuente: elaboración propia.

Los instrumentos investigativos elaborados se conformaron en sistema para responder a dos criterios fundamentales de caracterización del objeto de estudio: 1) organización metodológica del proceso de formación doctoral; 2) necesidades básicas de aprendizaje de doctorantes y tutores en dependencia del ecosistema donde se trabaje (ciudad, rural, costero, montaña) por la necesidad de sustentar teórica y metodológicamente cada uno de los componentes que conformarán el modelo pedagógico que responda a las exigencias de cada ecosistema y de las características de los doctorantes y sus líneas investigativas, los cuales reflejan rasgos contextuales particulares para cada uno.

Tanto en Cuba como en México, la formación doctoral contempla las necesidades cognoscitivas de los doctorantes y la contextualización del lugar de procedencia de los temas de los doctorantes (S. A. Pastrana Corral y Z. Matos Columbié, 2019) y la exigencia social de transformar desde la ciencia para revertir los aspectos negativos encontrados.

\section{RESULTADOS Y DISCUSIÓN}

Los principales resultados obtenidos de los instrumentos aplicados revelaron que: aunque aparecen muy bien delimitados en los programas de formación doctoral los objetivos y la metodología de organización del proceso formativo, es necesario ajustar las líneas y los temas a las principales problemáticas de la realidad objetiva y contextual para que entronice con el desarrollo local. Para lo cual se explicita la siguiente tabla.

Tabla 2. Resultados obtenidos de instrumentos aplicados a formación doctoral.

\begin{tabular}{|c|c|}
\hline Los doctorantes & Los profesores y tutores \\
\hline $\begin{array}{l}\text { Necesidad de intensificar la autopre- } \\
\text { paración profesional personal ( } 32= \\
100 \%) \text {; } \\
\text { Necesidad de utilizar un lenguaje } \\
\text { común y una misma línea de pen- } \\
\text { samiento en la determinación de los } \\
\text { temas y la orientación de la tesis ( } 28 \\
=87.5 \%) \\
\text { La posibilidad de integrar proyectos. } \\
(28=87.5 \%) \\
\text { La necesidad de realizar talleres con- } \\
\text { tinuos para la orientación y guía en el } \\
\text { trabajo de tesis ( } 31=96.9 \%) \\
\text { Los tutores y otros profesores deben } \\
\text { dominar las características de la co- } \\
\text { munidad donde se efectúa la investi- } \\
\text { gación }(28=87.5 \%) \\
\text { La necesidad de integrar las clases, la } \\
\text { tarea de investigación del proyecto, } \\
\text { la orientación de la tesis, y de los artí- } \\
\text { culos }(31=96.9 \%)\end{array}$ & $\begin{array}{l}\text { La necesidad de determinar con } \\
\text { más precisión y claridad: la re- } \\
\text { lación necesidades sociales con- } \\
\text { textualizadas; proyecto inves- } \\
\text { tigativo, línea de investigación; } \\
\text { tesis doctoral. ( } 31=96.9 \% \text { ) } \\
\text { La necesidad de construir un } \\
\text { modelo pedagógico de forma- } \\
\text { ción doctoral contextualizado a } \\
\text { las condiciones de los ecosiste- } \\
\text { mas frágiles en ambos países ( } 31 \\
=96.9 \% \text { ) } \\
\text { Potenciar la realización de ta- } \\
\text { lleres con los directivos territo- } \\
\text { riales y de la universidad, (32 = } \\
100 \% \text { ) } \\
\text { Realizar talleres postdoctorales } \\
\text { para debatir, analizar y llegar a } \\
\text { consenso sobre las buenas prác- } \\
\text { ticas para la formación doctoral } \\
\text { en condiciones las concretas de } \\
\text { las universidades de Guantána- } \\
\text { mo (Cuba) y Sonora (México). } \\
\text { Diseñar las vías de generaliza- } \\
\text { ción de los resultados a las co- } \\
\text { munidades de los ecosistemas } \\
\text { donde se trabajará después. (31 } \\
=96.9 \% \text { ) }\end{array}$ \\
\hline
\end{tabular}

Fuente: elaboración propia

A continuación, se explicita la esencia del modelo pedagógico de formación doctoral y su impacto académicosocial para el desarrollo local en la actualidad, se construye desde una perspectiva estratégica, por eso se destaca entre sus componentes estructurales y funcionales los que se mencionan en la figura 1 . 
Figura 1. Componentes del modelo pedagógico de formación doctoral

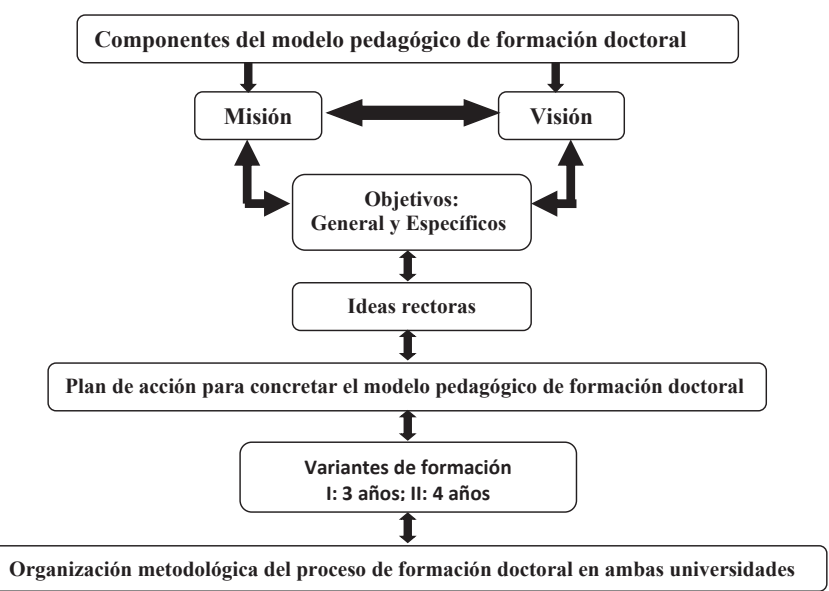

Fuente. Elaboración propia.

A continuación se explicita cada componente:

MISIÓN. Generar y socializar los conocimientos científicotecnológicos, la innovación de tecnologías en función del desarrollo local sostenible de los diferentes municipios de la provincia Guantánamo y del estado de Sonora.

\section{VISIÓN}

1. Los resultados investigativos que se produzcan de cada tesis dan respuestas a las crecientes demandas y exigencias del desarrollo local endógeno de los ecosistemas frágiles a partir de un diagnóstico integral para lograr impactos en diferentes aristas en las comunidades de los municipios.

2. Crece la fuerza profesional y científico-técnica de las universidades y los municipios para dar solución a la demanda con profesionales de perfil amplio, integrales y competentes para el desarrollo local sostenible y sustanteble.

3. La informatización garantiza el acceso de los profesores y doctorantes al conocimiento más actualizado de las ciencias, la educación y la tecnología, contextualizando el uso de herramientas digitales acorde a las plataformas y redes de las universidades y los ecosistemas frágiles.

4. Se fortalece la excelencia académica y profesional de los colectivos pedagógicos de cada universidad como consecuencia del incremento pertinente del número de doctores en Ciencias.

OBJETIVO GENERAL. Fortalecer el proceso de formación doctoral, en líneas priorizadas de los ecosistemas frágiles, con la colaboración directa de las universidades de Guantánamo y Autónoma de Sonora, para elevar el impacto sociocultural y ambiental de las ciencias, en correspondencia con la proyección estratégica de desarrollo local sostenible.

\section{OBJETIVOS ESPECÍFICOS:}

1. Fortalecer el intercambio científico permanente entre profesores, tutores, especialistas y con la red de la Universidad de Guantánamo, UNISON y sus revistas para la formación doctoral y postdoctoral mediante diferentes vías.

2. Contribuir al desarrollo de la teoría y mejorar la práctica educativa, social y productiva en los ecosistemas frágiles con la aplicación de los resultados derivados de la actividad científica investigativa por proyectos $\mathrm{i}+\mathrm{D}+\mathrm{I}$ y las tesis doctorales que respondan a las necesidades del país, y los ecosistemas frágiles del municipio como respuesta a las demandas de la Agenda 2030.

3. Desarrollar un claustro de excelencia en donde la formación doctoral constituya un proceso esencial para lograr impactos de la ciencia y la innovación tecnológica en la educación, la sociedad y el medio ambiente, mediante proyectos de investigación, más desarrollo, más innovación $(i+\mathrm{D}+\mathrm{l})$, que generen nuevos conocimientos, ponencias para eventos científicos, premios y publicaciones en revistas de alto impacto.

4. Contribuir a la elevación sostenible de los niveles de producción científica, su internalización y comercialización que generen ahorro, transferencia tecnológica, metodologías, sustitución de importaciones a las universidades y comunidades.

Sobre la base de lo anterior se diseña un modelo pedagógico que toma como IDEAS RECTORAS las siguientes:

1. El cumplimiento del marco legal estipulada para la formación doctoral por la Comisión Nacional de Grados Científicos de la República de Cuba y los de México, así como las exigencias de la Universidad de Guantánamo y de la Universidad de Sonora en México.

2. Determinación de los proyectos de $I+D+i$, que sustentarán las tesis doctorales en dependencia de las líneas investigativas determinadas en cada programa doctoral y para cada ecosistema frágil, asi como utilizar y potenciar de los resultados en diferentes áreas del saber en la Universidad de Guantánamo, y de la Universidad de Sonora, México.

3. Determinación del banco de problemas a investigar (surgido de los diagnósticos en diferentes comunidades e información oficial) y los principales aportes por modalidades que serán ofrecidos para su socialización inmediata con los gobiernos comunitarios, municipales en aras de proyectar el desarrollo local en diferentes áreas socioeconómicas. 
4. Establecimiento de un sistema de trabajo que potencialice el trabajo del proyecto $\mathrm{I}+\mathrm{D}+\mathrm{i}$, en equipos investigativos por unidades muestrales que se constituyan en laboratorios de investigación para varios temas sistémicamente interrelacionados que enfocaran resultados para el desarrollo local de una comunidad determinada en lo social, económico, medioambiental, entre otros.

5. El proceso de formación doctoral debe garantizar la profesionalización de los investigadores y el incremento de las capacidades y competencias en su área de conocimientos, la innovación, la investigación en la solución de problemas científicos que potencien el desarrollo local.

6. Proyección de doctorados a partir de una perspectiva global en correspondencia con la política científicaeducacional y docente-investigativa de cada universidad en función del desarrollo local.

7. Análisis y valoración de las posibilidades concretas existen para desarrollar doctorados en la UG y la UNISON mediante las Becas convenidas entre ellas, potenciando los jóvenes profesionales.

8.Creación las condiciones financieras y materiales necesarias para facilitar el desarrollo de la formación doctoral en diferentes especialidades en ambas universidades.

9. La asesoría al gobierno y a las comunidades en los ecosistemas frágiles de los municipios en la gestión de proyectos $\mathrm{i}+\mathrm{D}+\mathrm{I}$ para el desarrollo endógeno desde el componente investigativo de los doctorantes en su vínculo con las prácticas preprofesionales y el servicio social comunitario en el acompañamiento a su desarrollo local.

Para el cumplimento de los elementos anteriores se evidencia un PLAN DE ACCIÓN que de manera general discurrirá según lo siguiente:

1. Revitalización del convenio entre ambas universidades para determinar las áreas que deben ser fortalecidas con la formación doctoral, elaborar los programas o asumir los que existan con la respectiva planta académica.

2. Determinación de las áreas y comunidades donde se incidirá en su desarrollo local en diferentes áreas a partir de la implementación de los proyectos de donde se derivarán las tesis, con equipos de investigación multidisciplinarios de ambas universidades.

3. Garantizar la preparación metodológica de la planta académica que conducirá el programa de doctorado con funciones específicas, determinadas en cada colectivo pedagógico para garantizar: lenguaje común, metodología de trabajo, tutores, entre otras.
4. Selección de los candidatos a cursar el programa una vez realizado el diagnóstico por parte del comité de doctorado designado por ambas universidades

5. Determinación del calendario escolar para el inicio y culminación de los programas doctorales, utilizando dos variantes para este modelo de formación. Variante I: tres (3) años para la cual debe poseer el título de máster o especialidad y Variante II: cuatro (4) años para los que no ostenten el título de máster, pero deben tener cursado el diplomado de preparación para la formación doctoral o cursar un diplomado análogo determinado por ambas universidades. $\mathbf{c}$

\section{CONCLUSIONES}

La Educación de Posgrado está considerada un proceso sustantivo del más alto nivel de la educación Superior, y se destaca aquí la formación doctoral en la que se preparan integralmente los cuadros científicos de las universidades, por eso es que los egresados deben desarrollar y poseer una preparación teórico, metodológica e investigativa a partir del desarrollo científico-tecnológico alcanzado, que le posibilite la transformación se su realidad en consonancia con el modelo social asumido.

La formación doctoral ocupa un lugar preponderante como área clave de desarrollo de la universidad, corresponde a ella organizar metodológicamente este proceso para garantizar la preparación de un doctor competente y preparado para lograr la transformación del estado inicial en un estado deseado de su problema de investigación que incida en el desarrollo local

\section{BIBLIOGRAFIA}

Duran Rill, R.; et al (2015). Escuela de formación doctoral de la Universidad de Guantánamo, una experiencia para el desarrollo local. Vol 15. Núm 52. Abril - Junio. http:// edusol.cug.co.cu/index.php/EduSol/issue/view/12. http://www.redalyc.org/html/4757/475747193009/

Hernández-Guzmán, L. y Nieto Gutiérrez, J. (2010). La formación doctoral en México, historia y situación actual. Revista Digital Universitaria 1 de mayo 2010 • Volumen 11 Número 5 • ISSN: 1067-6079. En http:// www.revista.unam.mx/vol.11/num5/art46/ int46.htm

Luna Serrano, E. y Cordero Arroyo, G. (2017). Contribuciones a la evaluación educativa desde la formación doctoral. Editorial Universitaria. ISBN: $9786077427087 \quad$ https://www.elsotano.com/libro/ contribuciones-a-la-evaluacion-educativa-desde-laformacion-doctoral_10518454 
Matos Columbié Z.; et al (2019). El laboratorio socioeducativo de montaña "Juan Carlos Gallego Torres" para el desarrollo estra-tégico de la formación doctoral en el ecosistema montañoso de Yateras, Guantánamo. Dpto. Formación de Grados científicos. Universidad de Guantánamo. (Inédito)

Robas-Díaz, F. E.; et al (2018). Experiencias y retos en la formación doctoral en Ciencias Pedagógicas en la Universidad de Guantánamo. Revista Edusol. Vol 18 número especial. http://200.14.55.139/index.php/ EduSol/issue/view/69
Rodríguez Castilla, L.; et al (2018). Experiencias internacionales en el desarrollo de habilidades informacionales en la formación doctoral. Revista Académica. Universidad de Costa Rica. Volumen 8, número 2: julio-diciembre 2018. DOI 10.15517/ECI. V8I2.30522. HTTPS://REVISTAS.UCR.AC.CR/INDEX. PHP/ECIENCIAS/ARTICLE/VIEW/30522

Pastrana Corral, S. (2018). El servicio social y el desarrollo social comunitario. Conferencia Magistral. Grupo de Formación Doctoral en Ciencias Pedagógicas. Escuela de Formación Doctoral. Universidad de Guantánamo. 